\title{
The Rapamycin derivative RAD inhibits mesangial cell migration through the CDK-inhibitor p27 ${ }^{\mathrm{KIP} 1}$
}

\author{
Christoph Daniel $^{1}$, Jeffrey Pippin ${ }^{2}$, Stuart J Shankland ${ }^{2}$ and Christian Hugo ${ }^{1}$ \\ ${ }^{1}$ Medizinische Klinik IV, Universität Erlangen-Nürnberg, Erlangen, Germany and ${ }^{2}$ Division of Nephrology, \\ Department of Medicine, University of Washington, Seattle, WA, USA
}

\begin{abstract}
The link between mesangial cell (MC) proliferation and migration during glomerular repair in the experimental mesangial proliferative glomerulonephritis suggests that cell cycle regulation and cell migration require similar pathways, such as cell cycle proteins. The immunosuppressant RAD inhibits mesangial cell (MC) proliferation via G1/S arrest. Moreover, RAD dramatically impairs glomerular healing in the anti-Thy1 model. We tested the hypothesis that RAD alters MC migration in vitro and that this effect was mediated by the CDK-inhibitors p21 CIP1 and $\mathrm{p} 27^{\mathrm{KIP}}$. Using a modified Boyden chamber in vitro migration assay, our results showed that RAD dose dependently (1-50 nM) inhibited fibronectin-induced chemotaxis in wild-type (wt) MC. RAD treatment prevented the decrease in $\mathrm{p} 27^{\mathrm{KIP} 1}$ induced by mitogenic growth factors, but had no effect on $\mathrm{P21} 1^{\mathrm{CP} 1}$ by Western blot analysis. The antimigratory effect of RAD in wt MC was substantially dependent on $\mathrm{P} 27^{\mathrm{KIP}}$, but not $\mathrm{P21}{ }^{\mathrm{CIP} 1}$, since the inhibitory effects of 1-10 nM RAD on MC migration were similar in p21 ${ }^{\mathrm{CP} 1}$ deficient and wild-type MC. The effect of RAD on MC migration was also examined in the anti-Thy1 model by BrdU-labeling of proliferating MC on day 3 that typically repopulate the glomerulus from the hilus. A control biopsy on day 3 was taken to define the starting point prior to the initiation of RAD (3 mg/kg or placebo). MC migration was determined on day 7 by measuring the distances of BrdU-labeled $\mathrm{MC}(\mathrm{OX}-7+/ \mathrm{BrdU}+$ cells) from the glomerular hilus using computerized morphometry. RAD significantly reduced the migratory response of BrdU-labeled MC compared to controls. We conclude that the immunosuppressant RAD effectively inhibits $\mathrm{MC}$ migration in vivo and in vitro thereby limiting the normal glomerular repair process after severe injury. Moreover, RAD-induced inhibition of MC migration in vitro is partially mediated by the CDK-inhibitor p27 ${ }^{\mathrm{KIP}}$, but not $221^{\mathrm{CIP}}$.

Laboratory Investigation (2004) 84, 588-596, advance online publication, 5 April 2004; doi:10.1038/labinvest.3700078
\end{abstract}

Keywords: glomerular migration; glomerular repopulation; anti-Thy1 model; RAD; CDK-inhibitors

Severe disruption of the mesangium (mesangiolysis) occurs in many human renal diseases such as IgA nephropathy, hemolytic uremic syndrome, mesangiocapillary glomerulonephritis, allograft rejection, and diabetic nephropathy. ${ }^{1}$ Incomplete repair of the mesangiolytic injury may be an important determinant of glomerular disease progression. Successful and complete glomerular repair after very severe disruption of the mesangium is demonstrated in experimental mesangial proliferative glomerulonephritis in the rat (anti-Thy1 nephritis), ${ }^{2}$ which serves as a useful model to study the mechanisms underlying these events. In the anti-Thy1 model,

Correspondence: C Hugo, Medizinische Klinik IV, Universität Erlangen-Nürnberg, Krankenhausstrasse 12, 91054 Erlangen, Germany.

E-mail: Christian.Hugo@rzmail.uni-erlangen.de

Received 31 August 2003; revised 3 December 2003; accepted 15 December 2003; published online 5 April 2004 complete restoration of the glomerular architecture after injury is followed by capillary angiogenesis, matrix remodeling, and simultaneous mesangial cell (MC) proliferation and migration. ${ }^{3-5}$ While recent studies in the anti-Thy1 model demonstrated that about $11 \%$ of the glomerular repopulation after injury is derived from bone marrow, the major part of the glomerular reconstitution originates in the kidney. ${ }^{4,6}$ Within 1-2 days, surviving 'reserve' cells from the extraglomerular and hilar region reconstitute the major part of the mesangium by a coordinated proliferative and migratory response which is complete by days $7-10 .^{3,4}$ Although glomerular cell migration and proliferation can be dissociated in certain experimental nephritis models ${ }^{5}$ and by blockade of certain cytokines such as $\mathrm{FGF}-2^{7}$ both mechanisms are required for glomerular repair/ regeneration and timely as well as spatially closely connected in the anti-Thy1 model. This apparent link between cell proliferation and migration 
suggests that cell cycle regulation and cell migration may be governed by similar pathways, such as cyclins, cyclin-dependent kinases (CDK), and in particular the CDK-inhibitors $\mathrm{p} 21^{\mathrm{CIP} 1}$ and $\mathrm{p} 27^{\mathrm{KIP} 1}$. We have previously shown that $\mathrm{p} 27^{\mathrm{KIP} 1}$ and $\mathrm{p} 21^{\mathrm{CIP} 1}$ expression is altered during glomerular repopulation in the anti-Thy1 model. ${ }^{8}$

The potent antiproliferative rapamycin derivative RAD is a new promising immunosuppressive drug for the treatment of organ and kidney transplantation, and more recently, has been shown to inhibit proliferation via G1/S arrest in inflammatory renal disease. ${ }^{9}$ Although RAD and rapamycin are generally considered to lack nephrotoxicity of the calcineurin inhibitors cyclosporine and tacrolimus, we (unpublished results) and others ${ }^{10}$ have recently demonstrated detrimental long term effects on the glomerular architecture when RAD therapy was given during the early proliferative/migratory phase of anti-Thy1 nephritis. Taken together, we hypothesized that RAD therapy may have a specific antimigratory effect in addition to being antiproliferative, which may contribute to its puzzling detrimental effects.

Accordingly, we designed studies to determine if RAD inhibits MC migration in vitro and in vivo during glomerular repopulation in addition to being antiproliferative, and asked if these effects required the CDK-inhibitors $\mathrm{p} 27^{\mathrm{KIP} 1}$ or $\mathrm{p} 21^{\mathrm{CIP} 1}$. Our results show that the immunosuppressant RAD effectively inhibits MC migration in vivo and in vitro and that this is partially mediated by $\mathrm{p} 27^{\mathrm{KIP}}$.

\section{Materials and methods}

\section{Cell Culture}

Cultured mouse mesangial cells (MMC) were isolated from $\mathrm{p} 21^{\mathrm{CIP} 1}{ }_{-}, \mathrm{p} 27^{\mathrm{KIP} 1}$-, and $\mathrm{p} 21^{\mathrm{CIP} 1} / \mathrm{p} 27^{\mathrm{KIP} 1}$-null or wild-type (wt) mice as previously described. ${ }^{11,12}$ MC were grown in DMEM containing $4.5 \mathrm{~g}$ glucose $/ \mathrm{l}^{-1}$ and supplemented with $10 \%$ fetal calf serum, L-glutamine, insulin, and penicillin/streptavidin.

\section{In Vitro Cell Migration Assay}

MC migration was investigated using a 48-wellmodified Boyden chamber and $0.1 \%$ gelatin-coated polyvinyl-pyrrolidone-free polycarbonate membranes with $8 \mu \mathrm{m}$ poresize (Neuroprobes, Inc. Gaithersburg, MD, USA). After $16 \mathrm{~h}$ preincubation with 0, 1, 10 and $50 \mathrm{nM}$ RAD (provided by Dr Schuler, Novartis Pharma AG Basel) 28000 mesangial cells/well were loaded into the upper chamber. In two initial experiments, RAD was administered to wt-MMC without preincubation. The bottom wells were filled with $40 \mu \mathrm{g} / \mathrm{ml}$ fibronectin, used as the chemoattractant, or $0.1 \% \mathrm{BSA}$, used as a negative control. The Boyden chamber was incubated for $5 \mathrm{~h}$ at $37^{\circ} \mathrm{C}$ and $5 \% \quad \mathrm{CO}_{2}$ to allow migration of cells through the membrane. Cells that had transmigrated were stained with Qick diff and three fields of vision per well were analyzed using phase-contrast microscopy at a $250 \times$ magnification. All assays were done in triplicate and each experiment was done a minimum of three times.

\section{Animal Model and Experimental Design}

The effect of RAD therapy on MC migration was also examined in the anti-Thy1 model of mesangioproliferative glomerulonephritis. Three days after disease induction proliferating MC repopulating the glomerulus from the hilus region, were labeled with i.p. BrdU-injection in 12 Sprague-Dawley rats. A kidney biopsy was taken simultaneously as previously described ${ }^{4}$ to define the baseline. Half the nephritic rats $(n=6)$ were given RAD by gavage at a concentration of $3 \mathrm{mg} / \mathrm{kg}$ bw and six rats were given placebo (only containing the solvent of RAD). MC migration was measured on day 7 of disease by measuring the distances of BrdU labeled MC (OX$7+/$ BrdU + cells) from the glomerular hilus using computerized morphometry, ${ }^{4,7}$ and we used the baseline biopsy obtained at day 3 (ie before the initiation of RAD) in these calculations. The exact protocol for our in vivo migration assay has been previously described in detail., ${ }^{3,4,7}$

Experimental mesangial proliferative glomerulonephritis (anti-Thy1 model) was induced by a single injection of the monoclonal anti-Thy1.1 antibody OX-7 $(0.35 \mathrm{ml} / 100 \mathrm{~g}$ bw) in male Sprague-Dawley rats $^{13}$ (Charles River, Sulzfeld, Germany). 54, 62, and $70 \mathrm{~h}$ after disease induction, intraperitoneal injections of $10 \mathrm{mg} / 100 \mathrm{~g}$ bw BrdU were given in all rats to label surviving mesangial reserve cells located at the extraglomerular mesangium and hilar region that just start to repopulate the glomerulus via proliferation (DNA synthesizing) and migration. Three injections were given to increase the number of labeled cells. At $2 \mathrm{~h}$ after the last BrdU injection (72 $\mathrm{h}$ after disease induction), a baseline renal survival biopsy was performed. Immediately after recovery from anesthesia either microemulsion preconcentrate as vehicle or RAD (Novartis Pharma AG, Basel, Switzerland) was given once daily orally by gavage at a dose of $3 \mathrm{mg} / \mathrm{kg}$ bw/day. The first two RAD doses were given as loading doses containing two-fold the amount of the later doses. All rats were killed 7 days after disease induction and renal tissues harvested.

To assess the effect of RAD therapy on the CDK inhibitor $\mathrm{p} 27^{\mathrm{KIP} 1}$ and total or phosphorylated $\mathrm{p} 70^{\mathrm{S} 6}$ kinase during glomerular repopulation, anti-Thy1 disease was induced in a second set of 12 rats, and RAD or placebo therapy was started on day 3 . Afterwards, a survival biopsy was done on day 4 and all rats were killed on day 7 . The percentage of 
glomerular cells staining positive for total or phosphorylated $\mathrm{p} 70^{\mathrm{S6}}$ kinase and the CDK inhibitor p2 $7^{\mathrm{KIP} 1}$ was evaluated as previously described.

In an additional third experiment, three nephritic rats each were given RAD or placebo as described in the second experiment, and protein from isolated glomeruli was extracted on day 4 for determination of the CDK-inhibitor $\mathrm{p} 27^{\mathrm{KIP} 1}$ and total and phosphorylated $\mathrm{p}^{\mathrm{S}} \mathrm{S}^{\mathrm{S}}$ kinase by Western blot analysis (see below).

\section{Immunohistochemistry}

Tissue for light microscopy was fixed in methyl Carnoy's solution, embedded in paraffine, and cut into $5 \mu \mathrm{m}$ sections for indirect immunoperoxidase staining as described elsewhere. ${ }^{4,13}$

MC were identified using an antibody against OX7 (Serotec, Ltd., Kidlington, Oxford, UK). ${ }^{4}$ The following additional antibodies were used in this study: Peroxidase-labeled Fab-fragments against BrdU (Boehringer, Mannheim, Germany); polyclonal rabbit anti-p21 ${ }^{\mathrm{CIP} 1}$ and anti-p27 ${ }^{\mathrm{KIP} 1}$ antibodies (Santa Cruz Biotechnology, Heidelberg, Germany); ${ }^{8}$ mouse monoclonal antibody against the total $\mathrm{p} 70^{\mathrm{s} 6}$ kinase as well as the phosphorylated $\mathrm{p}-\mathrm{p} 70^{\mathrm{S} 6}$ kinase (Santa Cruz). ${ }^{14}$ Negative controls for immunostaining included either deleting the primary antibody or substitution of the primary antibody with equivalent concentrations of an irrelevant murine $\mathrm{mAb}$ or preimmune rabbit IgG.

\section{Immunohistochemical Double Staining}

To determine the migratory response of the labeled, BrdU-positive MC during glomerular repopulation, double immunostaining for BrdU and OX-7, an MCspecific marker for MC was performed as previously described. ${ }^{4}$

The first primary antibody (OX-7) was incubated overnight at $4{ }^{\circ} \mathrm{C}$, followed sequentially by biotinylated rabbit anti-mouse IgG1 serum (Zymed, San Francisco, CA, USA), peroxidase conjugated Avidin D (Vector, Burlingame, CA, USA), color development with DAB without Nickel Chloride. Incubation in $3 \% \mathrm{H}_{2} \mathrm{O}_{2} /$ methanol for $20 \mathrm{~min}$ prevented any remaining peroxidase activity. Subsequently, the second primary antibody (Peroxidase labeled antiBrdU Fab-fragments, Boehringer, Mannheim, Germany) was applied overnight at $4^{\circ} \mathrm{C}$, followed by $\mathrm{DAB}$ with nickel as the second color reagent. Controls included omitting either the primary antibodies or replacing them by mouse serum, as well as omitting either the secondary antibodies.

\section{Morphometric Analysis of MC Migration}

Migration of labeled MC was quantitated using a computerized morphometry system (Visitron
Systems GmbH, Puchheim, Germany). The shortest distance from the border between extra- and intraglomerular mesangium (OX-7 staining) to any labeled OX-7 positive MC was measured. For each biopsy, more than 10 cross sections with an identifiable extraglomerular mesangium out of more than 100 cortical glomeruli were examined.

\section{Western Blot Analysis}

Protein was extracted either from isolated rat glomeruli by our standard sieving technique ${ }^{15}$ or from MMC cultures using $50 \mathrm{mM}$ Tris, $1 \%(\mathrm{v} / \mathrm{v})$ Nonidet P-40, $0.25(\mathrm{w} / \mathrm{v})$ sodium deoxycholate, $150 \mathrm{mM} \mathrm{NaCl}, 1 \mathrm{mM}$ EGTA, $1 \mathrm{mM} \mathrm{Na} \mathrm{VO}_{4}, 1 \mathrm{mM}$ $\mathrm{NaF}$, and proteinase inhibitor cocktail (Complete, Boehringer, Mannheim, Germany) as extraction buffer. To optimize solubilization, extracts were sonified for $30 \mathrm{~s}$ at $50 \%$ power and $50 \%$ duty cycle using a Sonoplus HD70 (Bandelin, Berlin, Germany). A measure of $30 \mu \mathrm{g}$ of protein was resolved in sodium dodecyl sulfate-polyacrylamide gel electrophoresis, and proteins were transferred semidry to a nitrocellulose membrane for detection of p21 $1^{\mathrm{CIP} 1}, \mathrm{p} 27^{\mathrm{KIP} 1}, \mathrm{P} 70^{\mathrm{S} 6}$ kinase or phosphorylated $\mathrm{P}^{\mathrm{S} 6}$ kinase protein by using specific antibodies (Santa Cruz). $\beta$-actin was detected simultaneously as loading control using an antibody from Abcam, Cambridge, UK. For evaluation of densitometry AIDA 2.1 software (Raytest Isotopenmeßgeräte $\mathrm{GmbH}$, Straubenhard, Germany) was used.

\section{Statistical Analysis}

All values are expressed as mean s.d. Statistical significance (defined as a $P$ of less than 0.05 ) was evaluated using the Student's $t$-test or one-way analysis of variance with modified $t$-test using the Bonferroni method.

\section{Results}

\section{In Vitro Studies}

$R A D$ inhibits $M C$ migration in vitro

We used a 48-well modified Boyden chamber in vitro migration assay to determine the effects of $\mathrm{RAD}$ on migration. Pretreatment with RAD for $16 \mathrm{~h}$ inhibited fibronectin-induced chemotaxis in a dose-dependent matter in wt mouse MC (wtMMC). Our results showed that RAD reduced wtMMC migration by $20 \%$ at $1 \mathrm{nM}, 50 \%$ at $10 \mathrm{nM}$, and $75 \%$ at $50 \mathrm{nM}$ concentration, respectively, compared to placebo $(P<0.05)$ (Figure 1). Similar results were obtained in a minimum of three experiments. Significant inhibition of wt-MMC migration was also seen when no RAD pretreatment occurred and RAD was given only in the Boyden chamber during the migration time of $5 \mathrm{~h}(30 \%$ at $50 \mathrm{nM})$. Nevertheless, since RAD induced inhibition of cell 


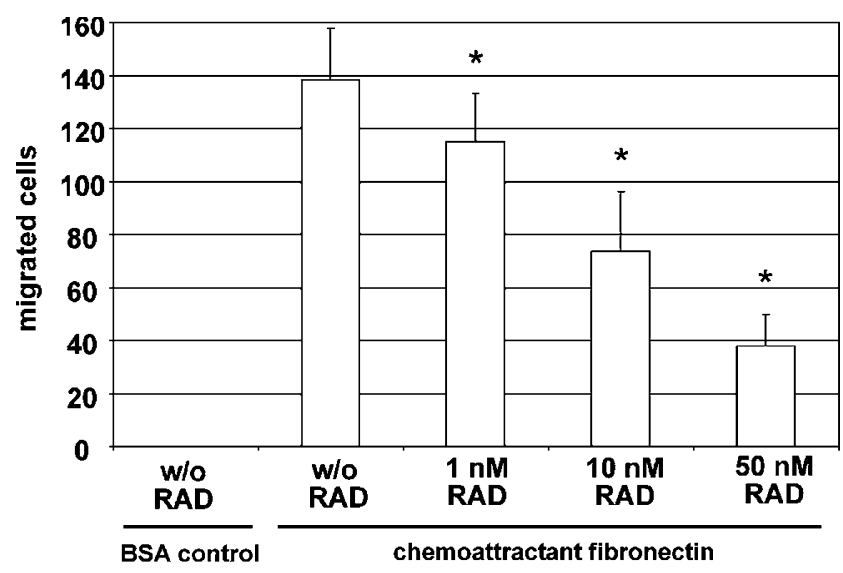

Figure 1 RAD inhibits MC migration in vitro. Using a 48-well modified Boyden chamber in vitro migration assay, pretreatment with RAD for $16 \mathrm{~h}$ inhibited fibronectin-induced chemotaxis in a dose-dependent matter in wt-MMC. The asterisk $\left({ }^{*}\right)$ indicates significant changes vs untreated controls $(P<0.05)$.

migration was much stronger after preincubation of MMC for $16 \mathrm{~h}$, all following experiments were done with RAD pretreatment.

$R A D$ regulates $p 27^{K I P 1}$, but not $p 21^{C I P 1}$ protein in $M C$ in vitro

We next determined, if RAD alters the levels of specific CDK-inhibitors. Exposing MMC to RAD differentially affected the protein levels of the CDK-inhibitors $\mathrm{p} 21^{\mathrm{CIP} 1}$ and $\mathrm{p} 27^{\mathrm{KIP} 1}$ measured by Western blot analysis in wt-MMC. As we showed previously, p27 ${ }^{\mathrm{KIP} 1}$ was very abundant in growtharrested wt-MMC. In contrast, p27 levels were reduced following stimulation with $10 \%$ FCS, a source of growth factors (Figure 2). Our results showed that 10 and $20 \mathrm{nM}$ of RAD significantly prevented the decrease in $\mathrm{p} 27^{\mathrm{KIP} 1}$ induced by growth factor stimulation (10\% FCS) (Figure 2). RAD at a $100 \mathrm{nM}$ concentration was less effective in preventing the FCS-induced decrease of p27 $7^{\mathrm{KIP} 1}$ (Figure 2). In contrast to $\mathrm{p} 27^{\mathrm{KIP} 1}$, protein levels of $\mathrm{p} 21^{\mathrm{CIP} 1}$ were not altered by FCS or RAD treatment (Figure 2). Taken together, these results show that RAD has a differential effect on CDK-inhibitor expression.

$R A D$-induced inhibition of MC migration is $p 27^{K I P 1}$, but not $p 21^{\text {CIP1 }}$ dependent

The role of $\mathrm{p} 21^{\mathrm{CIP} 1}$ and $\mathrm{p} 27^{\mathrm{KIP} 1}$, alone and together, in RAD-induced inhibition of MC migration was assessed utilizing p21 ${ }^{\mathrm{CIP} 1}$-null, p27 ${ }^{\mathrm{KIP} 1}$-null, and $\mathrm{p} 21^{\mathrm{CIP} 1} / \mathrm{p} 27^{\mathrm{KIP} 1}$ double-null MMC. The inhibitory effects of 1-10 nM RAD on MC migration were similar in p21 ${ }^{\mathrm{CIP} 1}$-null and wt-MMC (Figure 3a). However, at a $50 \mathrm{nM}$ RAD concentration, the migratory response was significantly less inhibited in $\mathrm{p} 21^{\mathrm{CIP} 1}$-null compared to wt-MMC $(P<0.005)$ (Figure 3a). In contrast, the antimigratory effect of RAD in wt-MMC was substantially more dependent on $\mathrm{p} 27^{\mathrm{KIP} 1}$ than on $\mathrm{p} 21^{\mathrm{CIP} 1}$ as investigated via MMC from $\mathrm{p} 27^{\mathrm{KIP} 1}$ - and $\mathrm{p} 21^{\mathrm{CIP} 1} / \mathrm{p} 27^{\mathrm{KIP} 1}$-double-null mice.
FCS

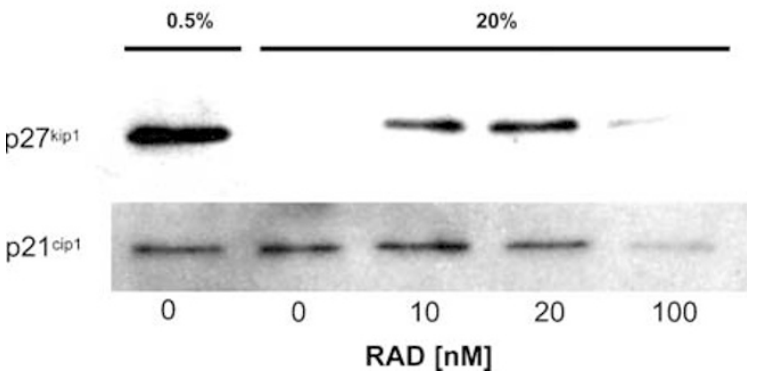

Figure 2 RAD regulates $\mathrm{p} 27^{\mathrm{KIP} 1}$, but not $\mathrm{p} 21^{\mathrm{CIP} 1}$ protein in $\mathrm{MC}$ in vitro. Regulation of the CDK-inhibitors $\mathrm{p} 21^{\mathrm{CIP} 1}$ and $\mathrm{p} 27^{\mathrm{KIP} 1}$ by RAD treatment was investigated in growth arrested $(0.5 \%$ FCS $)$ or growth factor stimulated (20\% FCS) wt MMC using Western blot analysis. While $\mathrm{p} 27^{\mathrm{KIP} 1}$ was highly expressed in growth-arrested wt-MMC, it was completely suppressed after stimulation with $20 \%$ FCS (Figure 2). RAD at a 10 and $20 \mathrm{nM}$, but hardly at a $100 \mathrm{nM}$ concentration markedly prevented the $\mathrm{p} 27^{\mathrm{KIP} 1}$ suppression by $20 \%$ FCS. In contrast, protein levels of $\mathrm{p} 21^{\mathrm{CIP} 1}$ appeared to be independent on FCS or RAD treatment.

In p27 $^{\mathrm{KIP} 1}$-null MMC, $1 \mathrm{nM}$ RAD had no effect and 10 and $50 \mathrm{nM}$ RAD reduced the number of migrating MMC only by 12 and $30 \%$, respectively, compared to inhibition of 47 and $72 \%$ in wt-MMC with similar concentrations $(P<0.001)$ (Figure 3a). In contrast, p21 ${ }^{\mathrm{CIP} 1}$-null MMC exhibited a 40\% (not significant compared to wt-MMC) and $46 \%(P<0.005$ compared to wt-MMC) decrease in the number of migrating cells when exposed to 10 and $50 \mathrm{nM}$ RAD, respectively (Figure 3a). In addition, when $\mathrm{p} 21^{\mathrm{CIP} 1} / \mathrm{p} 27^{\mathrm{KIP} 1}$ double-null MMC were compared to p27 ${ }^{\mathrm{KIP} 1}$-null $\mathrm{MMC}$, the migratory response under RAD therapy was similar $(P>0.05)$ (Figure $3 \mathrm{~b}$ ) suggesting that the additional lack of $\mathrm{p} 21^{\mathrm{CIP} 1}$ is not critical for migration of MMC.

\section{In Vivo Studies}

$R A D$ therapy inhibited MC migration in vivo We used the previously established in vivo migration assay to determine the effects of RAD on MC migration in the anti-Thy1 model of experimental mesangial proliferative glomerulonephritis. Figure 5 shows that RAD therapy reduced the migratory response of BrdU labeled MC by $75 \%$ compared to placebo-treated rats $(P<0.0005)$ (Figure 4$)$. In placebo-treated nephritic rats, labeled MC typically migrated $42.8 \pm 10.4 \mu \mathrm{m}$ (Figure $4 \mathrm{~b}$ ) from the hilus to the periphery of the glomerulus. In contrast, RAD significantly reduced migration of labeled $\mathrm{MC}$ in nephritic rats to $10.2 \pm 11.8 \mu \mathrm{m}$ (Figure 4c) within 4 days $(P<0.0005$ vs control). Therefore, this progressive migration of MCs towards the glomerular periphery occurs at a rate of $10.7 \pm 2.6 \mu \mathrm{m}$ per day in placebo-treated nephritic rats and only of $2.7 \pm 3.0 \mu \mathrm{m}$ per day during RAD treatment $(P<0.05)$ (Figure $4 \mathrm{~d})$. Taken together, these results 
592


Figure 3 RAD-induced inhibition of MC migration is $\mathrm{p} 27^{\mathrm{KIP} 1}$, but not $\mathrm{p} 21^{\mathrm{CIP} 1}$ dependent. RAD-induced inhibition of MC migration was assessed in p21 $1^{\mathrm{CIP} 1}$-and $\mathrm{p} 27^{\mathrm{KIP} 1}$-null MMC by a Boyden chamber assay using fibronectin as the chemoattractants. MMC were pretreated with different concentrations $(1,10,50 \mathrm{nM}) \mathrm{RAD}$ for $19 \mathrm{~h}$. The asterisk $\left(^{*}\right)$ indicates significant changes vs wt-MMC $(P<0.005)$. Since the antimigratory effect of RAD in wt-MC was substantially more dependent on $\mathrm{p} 27^{\mathrm{KIP} 1}$ than on $\mathrm{p} 21^{\mathrm{CIP} 1}$, the RAD dependency at different concentrations $(1,10,50 \mathrm{nM})$ of the migratory response was also compared in cultured MMC from p2 $7^{\mathrm{KIP} 1}$-null vs $\mathrm{p} 21^{\mathrm{CIP} 1} / \mathrm{p} 27^{\mathrm{KIP} 1}$ double-null mice (Figure $4 \mathrm{~b}$ ) and no significant change was found $(P>0.05)$.

show that RAD treatment markedly inhibits MC migration in the anti-Thy 1 model in vivo.

In anti-Thy1 nephritis, RAD therapy did not induce p $27^{K I P 1}$ protein expression, but prevented phosphorylation of the $p 70^{\text {S6 }}$ kinase

Since RAD prevented MC migration in vitro via p27 ${ }^{\mathrm{KIP} 1}$, we examined, whether RAD treatment would also upregulate $\mathrm{p} 27^{\mathrm{KIP} 1}$ in anti-Thy1 nephritis. To investigate the regulation of the CDK inhibitor p27 ${ }^{\mathrm{KIP} 1}$ by RAD therapy in the anti-Thy1 model in vivo, total glomerular cell number and the number of nuclei per glomerulus that stained positive for $\mathrm{p} 27^{\mathrm{KIP} 1}$ was assessed in a blinded manner. The percentage of $\mathrm{p} 27^{\mathrm{KIP} 1}$-positive cells per total glomerular cell number was calculated in placebo- and RAD-treated nephritic rats. Despite regulation of $\mathrm{p} 27^{\mathrm{KIP} 1}$ by $\mathrm{RAD}$ in $\mathrm{MC}$ in culture, Figure 6a shows that RAD therapy did not change the percentage of $\mathrm{p} 27^{\mathrm{KIP} 1}$-positive glomerular cells (Figure 5a) on day 4 or 7 in anti-Thy1 nephritis in vivo. This result was confirmed by Western blot analysis for $\mathrm{p} 27^{\mathrm{KIP} 1}$ using glomerular extracts from
anti-Thy1 diseased rats on day 4 with/without RAD treatment (Figure 6).

To demonstrate that RAD was nevertheless acting via its usual known signal transduction molecules in vivo in our model, the expression and phosphorylation of the $\mathrm{p} 70^{\mathrm{s} 6}$ kinase were investigated by immunostaining and Western blot analysis. In antiThy1 nephritis, RAD therapy selectively reduced the percentage of positive cells for the phosphorylated form of $\mathrm{p} 70^{\mathrm{S} 6}$ kinase per total glomerular cell number (Figure $5 \mathrm{~b}$ ), but did not affect the percentage of glomerular p70 $0^{\mathrm{s} 6}$ kinase-positive cells (Figure 5c). This result was also confirmed, when expression and phosphorylation of $\mathrm{p} 70^{\mathrm{S6}}$ kinase were examined on day 4 using Western blot analysis (Figure 6).

\section{Discussion}

The antiproliferative and immunosuppressive RAD used in kidney transplantation, inflammatory renal disease, and vasculitis ${ }^{9}$ has recently been studied in experimental mesangial proliferative glomerulonephritis (anti-Thy1 model). ${ }^{10}$ However, despite being antiproliferative, which one would expect to be beneficial as it is with other antiproliferative agents, ${ }^{15-18}$ RAD prevents glomerular repair in antiThy1 nephritis, leading to worsening renal function.

Repair of mesangiolysis requires both MC migration and proliferation. ${ }^{3,4}$ The novel results of the current study demonstrate that just in addition to inhibiting proliferation of $\mathrm{MC}$ in vitro and in vivo, ${ }^{10,19}$ RAD also dramatically prevents the migratory response of $\mathrm{MC}$ in vitro and in vivo. Previous studies have shown that following $\mathrm{MC}$ injury in the anti-Thy1 model, the surviving 'reserve MC' in the extraglomerular mesangium and hilar region are attracted to migrate to the periphery of the glomerulus in a coordinated way to repopulate the glomeruli. ${ }^{3,4}$ Therefore, by inhibiting this repairative response to injury, RAD prevents the healing phase of mesangiolysis. A similar effect has been observed, when conventional ${ }^{16}$ and nonanticoagulant ${ }^{20}$ heparin treatment was applied in the anti-Thy1 model in vivo. While no in vivo data regarding $\mathrm{MC}$ migration are available, the antiproliferative agent heparin also induces inhibition of MC migration in vitro. ${ }^{21}$ In contrast to the adverse RAD effects seen in the anti-Thy1 model, the transient prolongation of the mesangiolytic phase during heparin treatment did completely resolve within 40 days and even led to long-term beneficial consequences regarding renal functional and structural parameters after 120 days. ${ }^{16,20}$ It also has to be considered that the degree of cellular effects regarding proliferation/ migration may be very different with RAD vs heparin treatment.

If migration and proliferation of the repopulating MC is markedly prevented, the mesangium is limited to the perihilar area of the glomerular tuft and an important counterpart for stabilizing the 




Figure 4 RAD therapy inhibited MC migration in the anti-Thy1 model in vivo. Using the in vivo migration system as described in Materials and methods, the distance of BrdU labeled MC from the hilus to the periphery of the glomerulus on day 7 of anti-Thy1 disease was compared to their distance at the starting point on day 3. Double staining for OX-7 (marker for MC, brown) and BrdU (black) demonstrates the labeling of DNA synthesizing MC at the starting point of this assay (on day 3) close to the hilus (a). As shown before, many BrdU-labeled MC could be detected out in the glomerular periphery in placebo-treated anti-Thy1 diseased animals on day 7 (b). In RAD-treated anti-Thy1 rats, labeled MC stayed much closer to the hilar region indicating a defect in the migratory response (c). Quantitation of this assay by computerized morphometry demonstrates that on average, labeled MC in placebo treated rats migrated $42.8 \pm 10.4 \mu \mathrm{m}(\mathbf{d})$, while labeled MC in RAD-treated diseased rats only traveled $10.2 \pm 11.8 \mu \mathrm{m}(\mathbf{d})$ within 4 days. The asterisk $\left({ }^{*}\right)$ indicates significant changes vs placebo-treated rats $(P<0.05)$.

forces of the glomerular tuft ${ }^{22}$ in the periphery may be completely missing. This suggests that the early postmesangiolytic phase is vulnerable, for the later outcome critical phase, where the decision between a successful glomerular healing reaction or a chronic progressive time course is made.

Previous studies in experimental mesangioproliferative glomerulonephritis models have demon- 


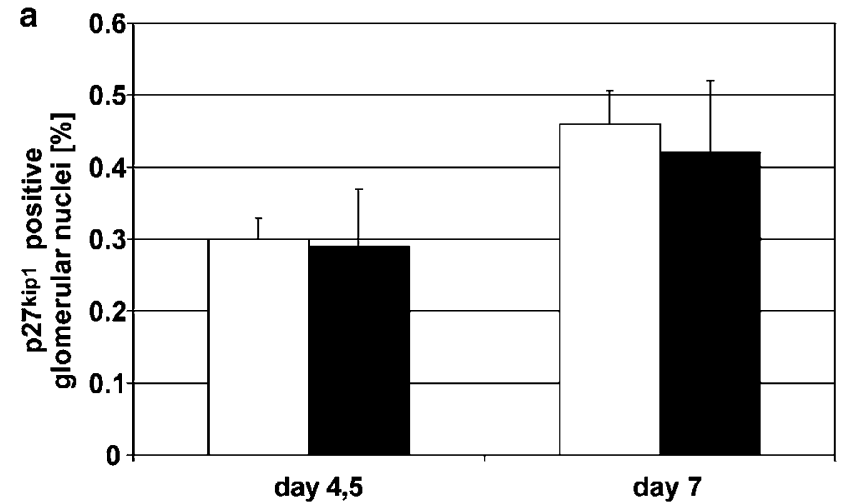

b

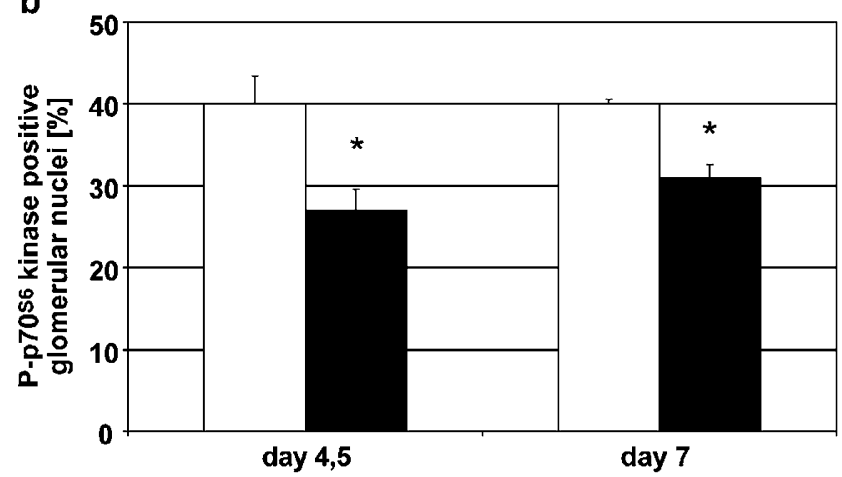

C

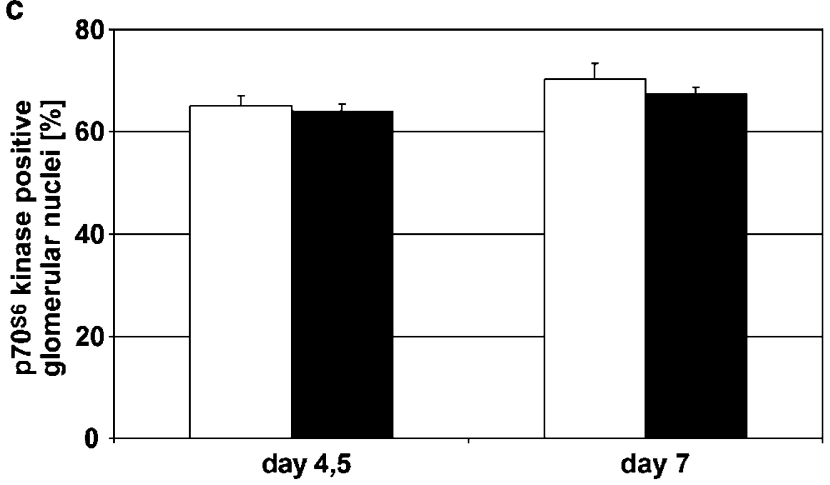

Figure 5 RAD therapy did not alter p27 $7^{\mathrm{KIP} 1}$ protein, but phosphorylation of the $\mathrm{p} 70^{\mathrm{S} 6}$ kinase in vivo (immunostaining). The glomerular percentage of nuclei that stained positive for p2 $7^{\text {KIP1 }}$ in RAD or placebo-treated anti-Thy1 rats was assessed on days 4 and 7 (a). The expression and phosphorylation of the p70 ${ }^{\mathrm{S} 6}$ kinase, a well-known RAD/rapamycin target protein, was further investigated in the same tissues. The glomerular percentage of positive cells for the phosphorylated (b) and total (c) form of the p70 ${ }^{\mathrm{S} 6}$ kinase was evaluated. The asterisk $\left({ }^{*}\right)$ indicates significant changes vs placebo-treated rats $(P<0.05)$.

strated that MC proliferation and migration can be dissociated..$^{5,7}$ Blocking of FGF-2 in the anti-Thy1 model resulted in inhibition of mesangial cell proliferation without significant effects on cell migration. ${ }^{7}$ In the Habu snake venom glomerulonephritis model, MC migrated during the first $24 \mathrm{~h}$ after disease induction without significant proliferation. ${ }^{5}$ Therefore, inhibition of cell proliferation is not linked with simultaneous inhibition of migra-
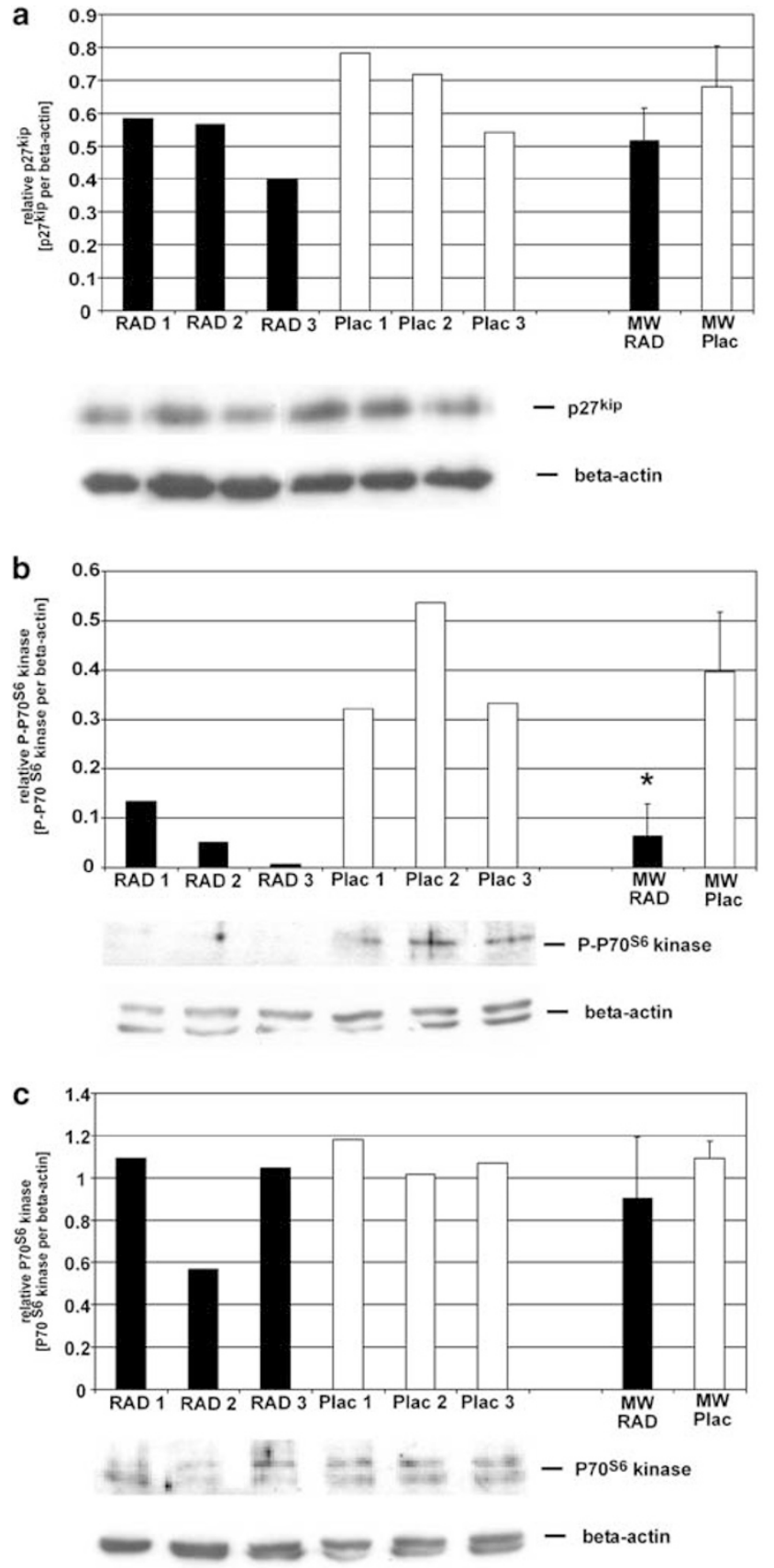

Figure 6 RAD therapy did not alter $\mathrm{p} 27^{\mathrm{KIP} 1}$ protein, but phosphorylation of the $\mathrm{p}^{\mathrm{S} 6}$ kinase in vivo (Western blot analysis). Independently, glomerular extracts anti-Thy1 diseased rats on day 4 with/without RAD treatment starting on day 3 were evaluated regarding $\mathrm{p} 27^{\mathrm{KIP} 1}$ and the expression and phosphorylation of the p70 ${ }^{\text {S6 }}$ kinase by Western blot analysis. The asterisk $\left({ }^{*}\right)$ indicates significant changes vs placebo-treated rats $(P<0.05)$.

tion in a general fashion. ${ }^{7}$ Nevertheless, many growth factors have the potential to act as both mitogens and chemoattractants. ${ }^{23}$ The apparent link of cellular proliferation and migration during glomerular repopulation in the anti-Thy1 model suggests that cell cycle regulation and cell migration may be regulated by similar pathways. In this study, 
we focused on the CDK-inhibitors p21 $1^{\mathrm{CIP} 1}$ and p2 $7^{\mathrm{KIP} 1}$, because these have been previously shown to be regulated during glomerular repopulation. ${ }^{8,24}$ Utilizing cultured MC from wt, p21 ${ }^{\mathrm{CIP} 1}$-null, p27 ${ }^{\mathrm{KIP} 1}$ null, and $\mathrm{p} 21^{\mathrm{CIP} 1} / \mathrm{p} 27^{\mathrm{KIP} 1}$-double-null mice, we demonstrated that the CDK-inhibitor $\mathrm{p} 27^{\mathrm{KIP} 1}$, but not p21 ${ }^{\text {CIP1 }}$ plays a critical role in mediating the antimigratory properties of RAD in vitro. The lack of $\mathrm{p} 27^{\mathrm{KIP} 1}$ in MMC prevented the antimigratory effect of RAD up to a concentration of $10 \mathrm{nM}$, while higher RAD concentrations were able to inhibit MMC migration in Boyden chamber assays, but to a much lesser extent than in wt-MMC. These data are consistent with previous reports regarding vascular smooth muscle cell migration ${ }^{25,26}$ and suggest that at higher doses, p27 $7^{\mathrm{KIP} 1}$-independent actions of RAD can inhibit MC migration.

The role and RAD-dependent regulation of $\mathrm{p} 27^{\mathrm{KIP} 1}$ in MC migration may be clinically relevant, since $\mathrm{p} 27^{\mathrm{KIP} 1}$ is tightly regulated in glomerular disease. Therefore, we further investigated, whether p27 $7^{\mathrm{KIP} 1}$ levels are changed during RAD therapy in anti-Thy1 nephritis in vivo. Neither by determination of the percentage of $\mathrm{p} 27^{\mathrm{KIP} 1}$-positive cells per glomerular cross section nor by Western blot analysis of glomerular extracts, any RAD-dependent regulation of $\mathrm{p} 27^{\mathrm{KIP} 1}$ was found in anti-Thy1 nephritis, despite a marked effect on MC migration and on the phosphorylation of the $\mathrm{p} 70^{\mathrm{S} 6}$ kinase as another RAD signaling target. Nevertheless, this lack of $\mathrm{p} 27^{\mathrm{KIP} 1}$ regulation in glomeruli from anti-Thy1 nephritic rats does not completely rule out a role for $\mathrm{p} 27^{\mathrm{KIP} 1}$ in $\mathrm{MC}$ migration nor in RAD-induced prevention of MC migration. For the interpretation of the unchanged p27 ${ }^{\mathrm{KIP} 1}$ in vivo, it has to be considered that our 'glomerular' data reflect regulation of $\mathrm{p} 27^{\mathrm{KIP} 1}$ in all different cell populations (inflammatory cells, endothelial cells, podocytes and $\mathrm{MC}$ ), while only regulation for the repopulating mesangial fraction of glomerular cells is relevant. Alternatively, it is interesting that in cultured wtMMC, higher RAD doses (50-100 nM) were much less potent to prevent growth factor stimulated suppression of $\mathrm{p} 27^{\mathrm{KIP} 1}$ compared to lower doses around 10-20 nM (Figure 2) and its effects on MC migration became partially $\mathrm{p} 27^{\mathrm{KIP} 1}$ independent. This phenomenon in MC has also been found in vascular smooth muscle cells. ${ }^{26}$ Although in vivo and in vitro doses are difficult to compare, the in vivo dose of $3 \mathrm{mg} / \mathrm{kg}$ bw (and loading doses) RAD is certainly considered to be high. Nevertheless, in this specific in vivo experiment, p70 ${ }^{\text {S6k }}$ phosphorylation, but not expression as a classical RAD-signal transduction target was inhibited suggesting p27 ${ }^{\mathrm{KIP}}$ independent pathways in this model.

In conclusion, our results demonstrate that the immunosuppressant RAD dramatically inhibits MC migration in vitro and in vivo, which may be highly relevant for the inhibition of the glomerular healing process after severe mesangiolysis. Our in vitro results demonstrate that RAD-induced inhibition of MC migration is partially mediated by the CDKinhibitor p2 $7^{\mathrm{KIP} 1}$, but not p21 ${ }^{\mathrm{CIP} 1}$. Further specific blocking and overexpressing studies have to still define the role for $\mathrm{p} 27^{\mathrm{KIP} 1}$ in MC migration and in RAD-induced prevention of MC migration.

\section{Acknowledgements}

We would like to acknowledge Novartis Pharma AG Basel for providing RAD solution and RAD vehicle solution. This work was supported by the Deutsche Forschungsgemeinschaft, the SFB 423 'Nierenschäden: Pathogenese und regenerative Mechanismen' (TP B6) and the Interdisciplinary Center of Clinical Research (IZKF)-TP B30. The authors are indebted to Andrea Lüdke, Susanne Weber, Ulrike Goller, and Ossama Mohamed for their excellent technical help during these studies. Portions of this work was presented at the International Society of Nephrology annual meeting, Berlin, June 8-12, 2003 (NDT, 2003, Vol.18, Suppl. 4) and American Society of Nephrology annual meeting, San Diego, October 14-17, 2003 (J Am Soc Nephrol, 2003).

\section{References}

1 Morita T, Yamamoto T, Churg J. Mesangiolysis: an update. Am J Kidney Dis 1998;31:559-573.

2 Johnson RJ. The glomerular response to injury: progression or resolution? Kidney Int 1994;45: 1769-1782.

3 Hugo C, Pichler R, Gordon K, et al. The cytoskeletal linking proteins, moesin and radixin, are upregulated by platelet-derived growth factor, but not basic fibroblast growth factor in experimental mesangial proliferative glomerulonephritis. J Clin Invest 1996;97: 2499-2508.

4 Hugo C, Shankland SJ, Bowen-Pope DF, et al. Extraglomerular origin of the mesangial cell after injury. A new role of the juxtaglomerular apparatus. J Clin Invest 1997;100:786-794.

5 Barnes JL, Hevey KA, Hastings RR, et al. Mesangial cell migration precedes proliferation in Habu snake venominduced glomerular injury. Lab Invest 1994;70: 460-467.

6 Ito T, Suzuki A, Imai E, et al. Bone marrow is a reservoir of repopulating mesangial cells during glomerular remodeling. J Am Soc Nephrol 2001;12: 2625-2635.

7 Haseley LA, Hugo C, Reidy MA, et al. Dissociation of mesangial cell migration and proliferation in experimental glomerulonephritis. Kidney Int 1999;56: 964-972.

8 Shankland SJ, Hugo C, Coats SR, et al. Changes in cellcycle protein expression during experimental mesangial proliferative glomerulonephritis. Kidney Int 1996;50:1230-1239.

9 Schuler W, Sendrani R, Cottens S, et al. SDZ RAD, a new rapamycin derivative: pharmacological properties in vitro and in vivo. Transplantation 1997;64:36-42.

10 Daniel C, Ziswiler R, Frey B, et al. Proinflammatory effects in experimental mesangial proliferative glomerulo- 
nephritis of the immunosuppressive agent SDZ RAD, a rapamycin derivative. Exp Nephrol 2000;8:52-62.

11 Monkawa T, Hiromura K, Wolf G, et al. The hypertrophic effect of transforming growth factor-beta is reduced in the absence of cyclin-dependent kinaseinhibitors p21 and p27. J Am Soc Nephrol 2002; 13:1172-1178.

12 Hiromura K, Pippin JW, Fero ML, et al. Modulation of apoptosis by the cyclin-dependent kinase inhibitor p27(Kip1). J Clin Invest 1999;103:597-604.

13 Rost S, Daniel C, Schulze-Lohoff E, et al. P2 receptor antagonist PPADS inhibits mesangial cell proliferation in experimental mesangial proliferative glomerulonephritis. Kidney Int 2002;62:1659-1671.

14 Lieberthal W, Fuhro R, Andry CC, et al. Rapamycin impairs recovery from acute renal failure: role of cellcycle arrest and apoptosis of tubular cells. Am J Physiol Renal Physiol 2001;281:F693-F706.

15 Johnson RJ, Raines EW, Floege J, et al. Inhibition of mesangial cell proliferation and matrix expansion in glomerulonephritis in the rat by antibody to plateletderived growth factor. J Exp Med 1992;175:1413-1416.

16 Floege J, Eng E, Young BA, et al. Heparin suppresses mesangial cell proliferation and matrix expansion in experimental mesangioproliferative glomerulonephritis. Kidney Int 1993;43:369-380.

17 Floege J, Ostendorf T, Janssen U, et al. Novel approach to specific growth factor inhibition in vivo: antagonism of platelet-derived growth factor in glomerulonephritis by aptamers. Am J Pathol 1999;154:169-179.
18 Ziswiler R, Steinmann-Niggli K, Kappeler A, et al. Mycophenolic acid: a new approach to the therapy of experimental mesangial proliferative glomerulonephritis. J Am Soc Nephrol 1998;9:2055-2066.

19 Wang W, Chan YH, Lee W, et al. Effect of rapamycin and FK506 on mesangial cell proliferation. Transplant Proc 2001;33:1036-1037.

20 Burg M, Ostendorf T, Mooney A, et al. Treatment of experimental mesangioproliferative glomerulonephritis with non-anticoagulant heparin: therapeutic efficacy and safety. Lab Invest 1997;76:505-516.

21 Person JM, Lovett DH, Raugi GJ. Modulation of mesangial cell migration by extracellular matrix components. Inhibition by heparin like glycosaminoglycans. Am J Pathol 1988;133:609-614.

22 Kriz W, Elger M, Mundel P, et al. Structure-stabilizing forces in the glomerular tuft. J Am Soc Nephrol 1995;5:1731-1739.

23 Chicoine MR, Silbergeld DL, et al. Mitogens as motogens. J Neurooncol 1997;35:249-257.

24 Shankland SJ, Wolf G, et al. Cell cycle regulatory proteins in renal disease: role in hypertrophy, proliferation, and apoptosis. Am J Physiol Renal Physiol 2000;278:F515-F529.

25 Poon M, Marx SO, Gallo R, et al. Rapamycin inhibits vascular smooth muscle cell migration. J Clin Invest 1996;98:2277-2283.

26 Sun J, Marx SS, Chen HJ, et al. Role for p27(Kip1) in vascular smooth muscle cell migration. Circulation 2001;103:2967-2972. 